\title{
Effect of orientation and of shape similarity on perceptual grouping'
}

\author{
JACOB BECK \\ HARVARD UNIVERSITY
}

A method in which Os were asked to partition a pattern into two regions was used to investigate the perceptual grouping produced by changes in the orientation and shape of two-line figures. The results show that the judged similarity of the figures fails to predict the degree to which the figures form distinct perceptual groups. Grouping was most strongly influenced by differences in the orientation of the lines composing the figures. Crossing of lines making up the figures also affected grouping, but was less decisive than line orientation.

Beck (1966) used threshold judgments to investigate the perceptual grouping produced by changes in the orientation and shape of two-line figures. When either the orientation or shape of some of the figures in a pattern was changed without changing the direction of their component lines, grouping was not facilitated. However, if these changes altered the direction of the component lines from the vertical and horizontal to $45^{\circ}$ and $135^{\circ}$, perceptual grouping occurred more readily. This study also suggested that the similarity of figures was not a good predictor of perceptual grouping. The purpose of the present experiments was to extend the results of this earlier study by further examining (a) the effect of changes in orientation and shape on perceptual grouping, and (b) whether grouping can be predicted by the judged similarity of the figures.

\section{Method}

To determine the relative effectiveness of changes in orientation and shape on grouping, a method was used in which observers were asked to partition a pattern into two regions. The method is most easily illustrated by considering target $a$ in Fig. 1 . The pattern is made up of three sections; the observer is asked to divide the pattern into two regions, at the boundary where the most natural break occurs. The division in target a may be made at the transition from the backward $\underline{L}$ figures to the $T$ figures, or at the transition from the $\underline{T}$ figures to the slanted $\underline{T}$ figures. If a significant majority of Os divides the pattern at one boundary, this would indicate that the difference between the figures in the two adjacent regions at this boundary produces a stronger segregation.

Twelve patterns were used in the study. They measured approximately $11.25 \times 6.25 \mathrm{~cm}$. They were divided into thirds, each third having 36 identical figures. The arrangement of the figures was the same for each third and was also the same for all patterns. The figures were composed of two perpendicular lines, $6 \mathrm{~mm}$ in length; the lines were either vertical and horizontal, or rotated to $45^{\circ}$ and $135^{\circ}$. There was no distinct boundary between the figures of different shapes, and care was taken to control for the evenness of the boundaries. The patterns were made of the following figures:

(a) backward $\underline{L}, \underline{T}$, slanted $T$; (b) $\underline{V}, \underline{T}$, slanted $T$; (c) $\underline{V}, \underline{\text { slanted }} T, \underline{X}$; (d) $T, \underline{T}$ on its side, \pm ; (e) $T$, $\underline{T}$ on its side, backward $L$; $(f) \pm, \underline{x}, \underline{V} ;(g)$, slanted $\underline{T}$, V; (h) $T$, backward $L, \pm$; (i) $T$, slanted $T, \underline{X}$; (j), $\pm T$, slanted T; (k) T, backward L, T on its side; (1) $\underline{T}$, X, V. Figure 2 shows the three figures making up the twelve patterns.

a

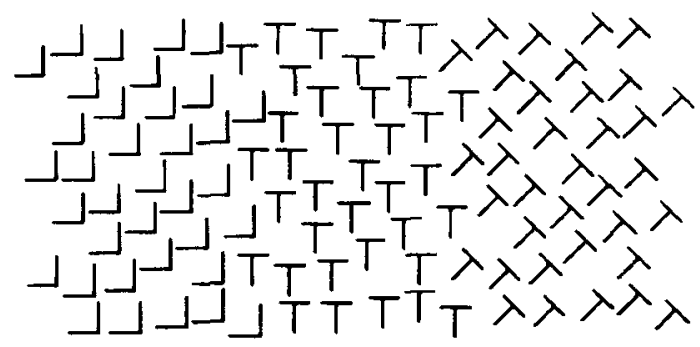

e
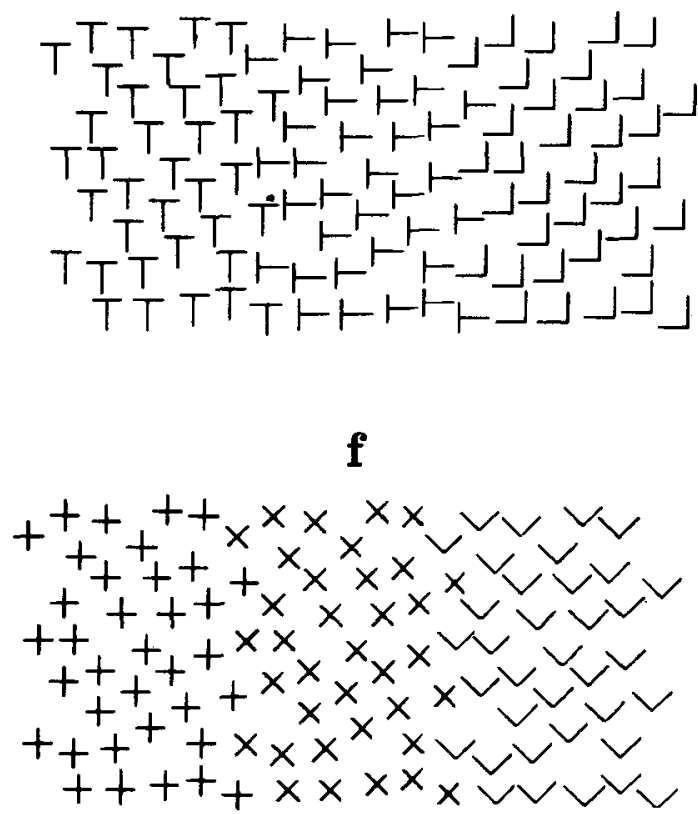

Fig. 1. Targets $a, e$ and $f$. Three of the 12 targets used in the experiment. The targets are shown in their upright position. 
Fifteen observers served in the experiment. The targets were placed on a stand $140.5 \mathrm{~cm}$ from the Os. They viewed the targets monocularly through an electrically controlled shutter which limited viewing time to $4 \mathrm{sec}$. A sample card was made up of three regions labeled $\mathrm{A}, \mathrm{B}$ and $\mathrm{C}$. The $\mathrm{O}$. were shown this demonstration card and told they would be shown similar targets with no sharp boundary between the sections. They were instructed to indicate at which boundary there was a more natural break in the pattern. The targets were presented both upright and inverted. Thus, there was a total of 24 stimuli. These were presented in a different irregular order to each $O$.

To determine the effect of figural similarity on grouping, 10 patterns from the previous experiment were scaled for similarity. On each of these patterns one of the regions was masked. The patterns were composed of the following figures:

(1) V, $T$; (2) $\underline{V}$, slanted $T$; (3) $\underline{T}, \underline{T}$ on its side; (4),$\pm \underline{X}$; (5) $\underline{T}$, slanted $T$; (6) $T$, backward $\mathrm{L}$; (7) slanted $T, \underline{X}$; (8),$\pm \underline{T}$; (9) backward $\underline{L}, \underline{T}$ on its side; (10) $\underline{X}, \underline{V}$. The Os were instructed to judge the similarity of the figures composing each region on a 10point scale; 0 indicated very little similarity, and 9 very great similarity. To familiarize the Os with the task, the 10 targets were shown to each $O$ in a preliminary trial. The patterns were again placed at $140.5 \mathrm{~cm}$ from the $\mathrm{Os}$ and viewing time was $4 \mathrm{sec}$. Ten Os, who had not participated in the previous experiment, made three judgments of each of the targets presented upright. The Os in both experiments were Harvard students who were paid to participate and were naive as to the purpose of the experiments.

\section{Results and Discussion}

Figure 2 presents the results of the grouping experiment. The order of the three figures as they appeared on each pattern when the target was upright are shown. Boundaries 1 and 2 always refer to the left and right boundaries of the pattern, respectively. What is boundary 1 when the target is upright becomes boundary 2 when the target is inverted. Thus, in target $a$, the separation between $\underline{T}$ and slanted $\underline{T}$ corresponds to boundary 2 when the target was upright, and to boundary 1 when the target was inverted. A comparison of the number of Os dividing the patterns at each boundary when the targets were upright and inverted shows that Os' judgments were very similar for both orientations of the pattern.

Examination of Fig. 2 indicates that (a) changes in the orientation of the figures do not produce two distinct groups unless the directions of the component lines of the figures in these two groups differ, and (b) when the component lines of the figures lie in the same direction, changes in shape of the figures do not produce grouping unless the change involves line crossing. On targets a, g, i and l, Os showed a highly significant preference for one boundary. In all these targets the figures with lines in the same direction are grouped together. When differences in slant were not present, as in targets $c, d$ and $h$, Os tended to group the figures with crossed lines separately. The results just fail to reach significance at the 5 percent level, except for target $d$ when presented upright. The relative effectiveness of slanted and crossed lines in producing grouping can be seen in targets $f, i$ and $j$. Targets $i$ and $j$ show that figures having the same line

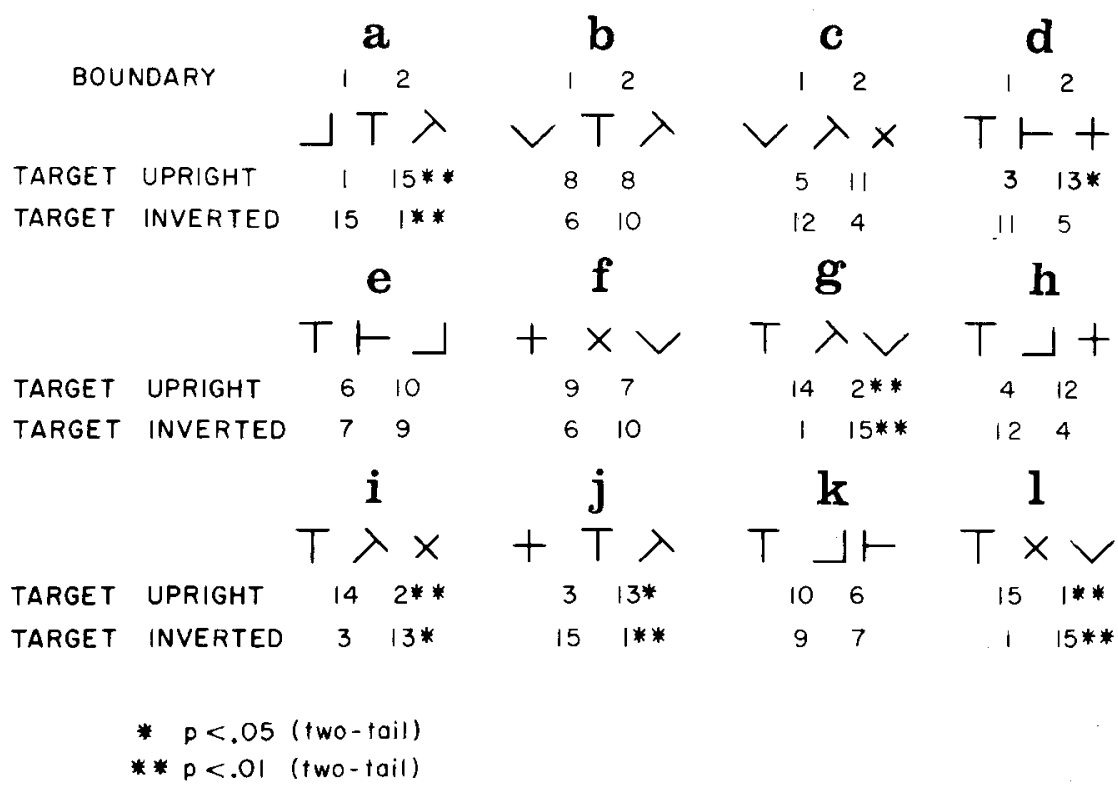

Fig. 2. Results of the Grouping Experiment. The order of the three figures as they appeared on each pattern when the target was upright is shown. Boundaries 1 and 2 always refer to the left and right boundaries of the pattern, respectively. What is boundary 1 when the target is upright becomes boundary 2 when the target is inverted. Thus, in target a the separation between $T$ and slanted $T$ corresponds to boundary 2 when the target was upright and to boundary 1 when the target was inverted. The numbers under each boundary denote the number of $0 \mathrm{~s}$ choosing that boundary. 


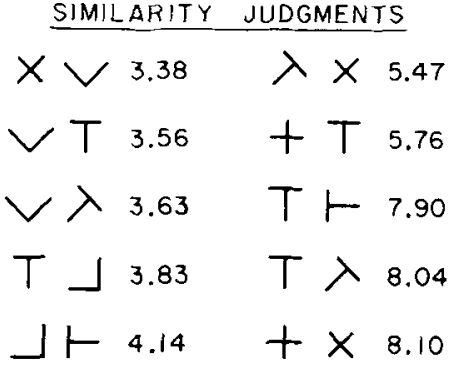

Fig. 3. Mean Similarity Ratings. The numbers denote the mean similarity ratings on a scale from 0 - very little similarity to 9 very great similarity averaged over the $100 \mathrm{~s}$.

orientation are grouped together, rather than figures with uncrossed lines. In target $f$, the responses are equally distributed between both boundaries. No marked preference occurs for either boundary in targets $b, e$ and $\mathrm{k}$. In all three patterns there is no grouping possible in terms of either line orientation or crossing.

Since rotating the figures from $90^{\circ}$ to $45^{\circ}$ changes their horizontal and vertical dimensions, it may be argued that the decisive variable for grouping is the difference in these dimensions rather than the slant of the lines. The results do not support this view. For example, the horizontal dimensions of the $\underline{X}$, slanted $\underline{T}$ and $\underline{V}$ are $4.24,6.36$ and $8.48 \mathrm{~mm}$, respectively. Since the $\underline{T}$ is $6 \mathrm{~mm}$ wide, on the basis of the width difference the boundary should be placed between $\mathrm{T}$ and slanted $\mathrm{T}$ in patterns $g$ and $i$, and between $T$ and $X$ in pattern 1 . Similar considerations will refute the argument that the vertical dimension or the area covered by the figure are the decisive variables.

What is the effect of figure similarity? Figure 3 presents the mean similarity ratings averaged over the 10 Os. As can be seen, the highest similarity ratings were for those figures which involve only a change in their orientation. The pairs of figures judged to be most similar are $\pm \underline{X}, \underline{T}$ slanted $\underline{T}$, and $\underline{T} \underline{T}$ on its side. The remaining scale values are lower and seem to depend on the arrangement of the lines composing the figures regardless of their orientation.

A comparison of Figs. 2 and 3 shows that the judged similarity of figures is a poor predictor of grouping. For example, $\underline{T}$ slanted $\mathbf{T}$ has a high rating of similarity, yet in patterns $a, b, g, i$ and $j$ they are never grouped together. $T$ T on its side also has a high rating of similarity, yet the results for pattern e show that there is no marked preference to group these two together. It is interesting to note that $\pm \underline{X}$ has the highest degree of similarity, and $\underline{\mathrm{X}} \mathrm{V}$ has the lowest similarity. This may account for the fact that in pattern $f$ Os' responses were not determined by line orientation.

In general, the results support the earlier findings (Beck, 1966) that it is necessary to examine the specific properties of figures to account for grouping. In the present study the most powerful variable for segregating the patterns was the difference in the orientation of the lines composing the figures. Crossing of the lines making up the figures also affects grouping, but is less decisive than line orientation. It is important to note that in the experiment the crossed figures were always composed of perpendicular lines that were symmetric about vertical, horizontal and inclined axes. Since crossing was associated with a high degree of symmetry, care is needed in generalizing about the relative importance of crossed lines.

\section{Reference}

Beck, J. Perceptual grouping produced by changes in orientation and shape. Science, 1966 , in press.

\section{Note}

1. This research was supported by NSF Grant GB-290I to the Psychological Laboratories, Harvard University, and by a Grant from the Carnegie Corporation to the Center for Cognitive Studies. Harvard University.

(Received in the Editorial Office July 18, 1966.) 